\title{
(Typo)graphies anarchistes. Où le genre révèle l'espace politique de la langue
}

Anarchist (typo)graphies. Where gender reveals the political space of the language

(Tipo)grafías anarchistes. Donde el género revela el espacio político de la lengua

Julie Abbou

\section{(2) OpenEdition}

\section{Journals}

Édition électronique

URL : https://journals.openedition.org/mots/22637

DOI : $10.4000 /$ mots. 22637

ISBN : 978-2-84788-893-5

ISSN : $1960-6001$

Éditeur

ENS Éditions

\section{Édition imprimée}

Date de publication : 9 mars 2017

Pagination : 53-72

ISBN : 978-2-84788-892-8

ISSN : 0243-6450

\section{Référence électronique}

Julie Abbou, « (Typo)graphies anarchistes. Où le genre révèle l'espace politique de la langue », Mots.

Les langages du politique [En ligne], 113 | 2017, mis en ligne le 09 mars 2019, consulté le 22 avril 2022

URL : http://journals.openedition.org/mots/22637 ; DOI : https://doi.org/10.4000/mots.22637

\section{(C) ENS Éditions}




\title{
(Typo)graphies anarchistes. Où le genre révèle l'espace politique de la langue
}

\author{
«La résistance et la subversion prennent souvent une \\ tournure qui serait impossible à l'oral.»
}

(Deborah Cameron, 1992, p. 217)

«La parenthèse ça a politiquement un sens qui me plaît pas.»

(Extrait d'entretien)

\section{La langue comme lieu politique, la graphie comme outil}

Le genre est un pot de miel : une fois qu'on plonge dans cette question, impossible d'en conclure à une solution ferme et définitive. Qui voudra questionner sa pertinence écrira des pages et des pages sur la question. Qui tentera de le gommer de son discours se prendra les pieds dans un enchevêtrement de possibilités typographiques, morphosyntaxiques et politiques. C'est que le genre est inextricablement linguistique et social à la fois ${ }^{1}$. Pour le questionner en tant que rapport de pouvoir, on peut donc l'interroger discursivement en tant que rapport social, mais également linguistiquement, en tant que catégorie grammaticalisée. Le genre est en effet la seule catégorie morphosyntaxique indexant des rapports de pouvoir. Les postures critiques sur le genre vont donc en permanence le soumettre à un double traitement, discursif et linguistique : on thématise le genre pour en faire la critique, on en fait le thème du discours, et on le rhématise pour continuer à en parler à propos d'autre chose : les manifestant.es.

Le marquage du genre dans le présent article suit les recommandations ortho-typographiques de la revue. En conséquence, les graphies de types «illes» n'y apparaissent pas, contrairement à la pratique de l'auteure. Cette précision permet d'illustrer, nous semble-t-il, toute la difficulté à tenir ensemble des normes ortho-typographiques et éditoriales cohérentes, et des pratiques linguistiques innovantes. [ndlr]

1. Le genre est simultanément linguistique et social en ce qui concerne la référence aux humains. Cependant, par la force métaphorique du genre, décrite par Roman Jakobson (1963), puis Claire Michard (1999), le genre linguistique des référents inanimés est également touché, c'est-à-dire réinterprété à la lumière du genre social. 
Les anarchistes, dont le cœur de la critique porte sur le pouvoir, s'attaquent au genre dans son versant linguistique. À côté de la critique théorique, en discours, du genre, ils vont développer une pratique linguistique du genre comme pratique politique. Il s'agit principalement d'un bousculement - ou d'un tumulte pour reprendre les mots d'Elsa Dorlin (2005) - de l'écriture. Si le genre est plus marqué à l'écrit qu'à l'oral, il y est également plus modifiable. La linéarité irréductible de l'oral s'efface à l'écrit devant la possibilité de rupture, de cohabitation de formes, et autres superpositions, autant de marges de manœuvre dans lesquelles la typographie va devenir un outil indispensable.

Je propose dans cet article d'explorer les formes graphiques de cette perturbation grammaticale à visée politique. Un corpus de textes anarchistes, dont on trouvera une présentation dans la section suivante, permettra de décrire les différentes (typo)graphies ${ }^{2}$ en usage. M'appuyant sur un second corpus d'entretiens menés avec des personnes utilisant ces graphies, je tenterai de montrer quels sont les enjeux politiques et langagiers qui se tiennent dans ces pratiques subversives de l'écrit. Enfin, je mettrai en perspective ce marquage en discutant de la place du discours dans la culture anarchiste, avant de développer une proposition d'analyse de ces pratiques comme rhématisation du genre, qui interroge le rapport émique/étique.

\section{Le double marquage de genre, une pratique linguistique contemporaine}

Les brochures anarchistes présentent des pratiques de perturbation graphique du genre. Avant de voir en quoi ces graphies relèvent de la perturbation ou du tumulte, en voici quelques exemples : émeutièrEs; auteurSEs; menteureuses; transgenderé-e-s.

Ces formes commencent à apparaître autour des années 1990. Un relevé systématique des brochures publiées sur le site 〈https://infokiosques.net/〉 (consulté le 19 janvier 2017), qui fournit une version numérique d'une très grande partie des brochures circulant dans les réseaux anarchistes, montre l'intensification de ces formes dans les années 2000, qui va de pair avec l'accroissement du nombre de brochures publiées. La figure ci-dessous (Fig. 1) fournit une représentation chronologique de l'apparition de cette perturbation linguistique du genre dans les brochures anarchistes. Les colonnes blanches représentent le nombre de brochures éditées et les colonnes grises le nombre de brochures dans lesquelles on trouve des marques de cette perturbation.

Cette pratique linguistique est donc constitutive de la production contemporaine de brochures anarchistes. Afin de la décrire, j'ai constitué un corpus de

2. Par (typo)graphie, je désigne des formes graphiques relevant soit de la morphosyntaxe, soit de la typographie, voire des deux. 
Fig. 1. Présence de la perturbation du genre dans les brochures anarchistes 1990-2008

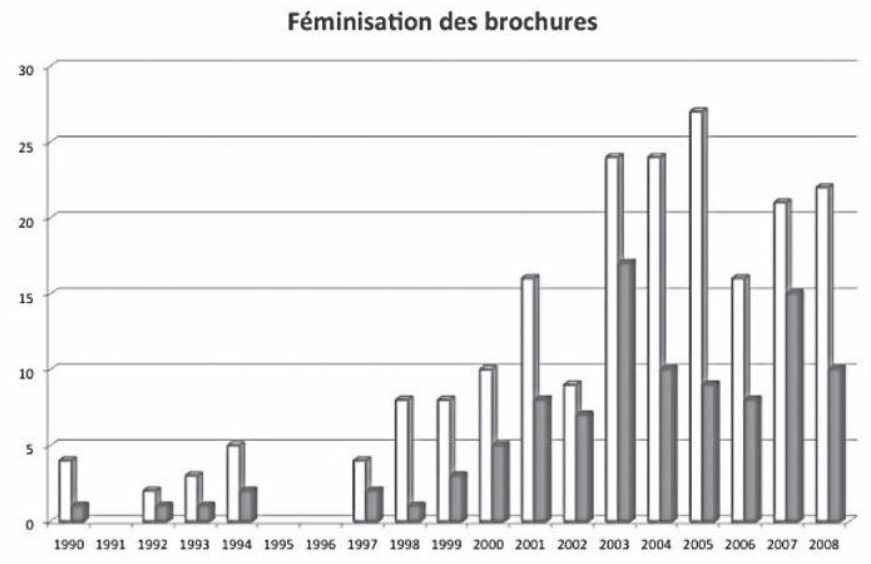

six brochures réunissant quinze textes 3 . Cet éventail de textes offre un panorama de la perturbation du genre dans les brochures libertaires. Les registres varient : pamphlet, témoignage, argumentaire, récit, manifeste, communiqué, appel, etc. Chacun de ces textes comporte des particularités qui vont plus ou moins influer sur le marquage du genre. Les autorités sont diverses également : collectives, pseudonymes ou anonymes.

Avant d'entrer dans la description du corpus, une mise au point terminologique est nécessaire. On regroupe généralement les modifications linguistiques concernant le genre sous le terme de féminisation (par exemple Houdebine, 1999). Certaines auteures choisissent de parler de désexisation (Dumais, 2004), de parité linguistique (Baider et al., 2007) ou encore de langage épicène (Moreau, 2001). Anthony Liddicoat (2011) note que ce qu'on nomme habituellement féminisation recouvre en fait trois types de pratiques. Le premier consiste en l'élaboration d'un langage des femmes, c'est-à-dire une féminisation littérale, au sens de mise au féminin, et non l'introduction du féminin au côté du masculin. C'est le cas de termes tels que femmage ou sororité, mais aussi des travaux d'écriture féminine produits par les féministes différentialistes des années 1970. La recherche linguistique de l'égalitarisme est le deuxième type, peut-être majoritaire aujourd'hui : il s'agit de réinjecter du féminin au côté du masculin, en agissant principalement sur les désignations génériques, pour rappeler la présence d'hommes et de femmes dans des groupes

3. Le corpus compte environ 38000 mots, disponible sur le site Ortolang : dépôt et diffusion / Banque de données parole et langage 〈http://sldr.org/sldroo0769〉 (consulté le 19 janvier 2017). La sélection du corpus a été réalisée dans l'idée d'offrir un panachage des pratiques et des thèmes abordés. Avec seulement six brochures, ce corpus ne peut offrir qu'une analyse qualitative qui n'a pas vocation à être représentative de l'ensemble de la production. 
ou fonctions habituellement désignés au masculin. Enfin, la perturbation, qui est au cœur de notre propos, a pour enjeu principal de faire apparaître les deux genres simultanément, dans une visée déconstructionniste. Les textes où apparaît cette intervention linguistique présentent donc - plus ou moins régulièrement - le masculin et le féminin simultanément, principalement pour les termes renvoyant aux humains. Puisqu'il est structurellement impossible en français d'éviter le genre, l'accolement des deux termes de l'opposition, permet d'annuler, ou du moins de montrer une volonté d'annulation de leurvaleur respective, comme $1+(-1)=0$, afin d'éliminer le genre comme trait distinctif. En effet, le genre repose fondamentalement sur un principe de distinction, en langue (dans les descriptions qu'en fait le structuralisme) comme en société (dans les descriptions qu'en fait le matérialisme). La présentation simultanée du masculin et du féminin est alors un moyen de contournement de la valeur distinctive du genre.

On pourrait discuter de la possibilité de parler de neutre, puisqu'il y a une volonté de neutraliser l'opposition de genre, mais il semble que ce soit dans ce corpus davantage une volonté de dire «absence/refus de la marque de genre », d'ôter le caractère catégoriel du genre lorsqu'il est motivé, plutôt qu'une «marque de l'absence de genre». C'est pourquoi je propose de parler de double marquage de genre. Cette dénomination permet également de faire apparaître le terme genre. Mais surtout elle permet d'attirer l'attention sur l'idée que la dualité du marquage est en premier lieu d'ordre formel (linguistique), alors que la binarité du genre social est, elle, refusée (ce que ne permet pas de faire apparaître le terme de féminisation).

Je parlerai par la suite de terme marqué. J'entends par là un signifiant dont le signifié désigne un animé (le plus souvent humain), qui porte les deux genres pour des raisons politiques de perturbation du genre : ajout d'éléments linguistiques féminins à un terme grammaticalement masculin (cas le plus fréquent) ou ajout d'éléments linguistiques masculins à un terme grammaticalement féminin.

\section{Description des formes (typo)graphiques utilisées pour un traitement politique du genre en langue}

Le premier constat qui émerge de l'observation détaillée des termes marqués du corpus est la diversité des procédés typographiques employés. En effet, pour marquer l'hétérogénéité du genre, de nombreux signes typographiques sont disponibles : le tiret, le point, le point médian, la majuscule, les parenthèses, les astérisques, le slash, etc. ainsi que l'évitement de signes typographiques par l'emploi de tournures lexicalement doubles (manifestantes et manifestants) ou formes longues (nombreuxses). On peut regrouper ces procé- 
dés selon les fonctionnements linguistiques du genre auxquels ils répondent: la cohabitation des formes (linéaire), l'alternance des formes (non-linéaire), et l'ajout du double marquage aux épicènes.

\section{Linéarité du masculin et du féminin}

Pour un certain nombre de termes, le genre va fonctionner de manière linéaire, c'est-à-dire sur un modèle forme courte/forme longue, avec généralement le masculin comme forme courte et le féminin comme forme longue 4 .

Dans ce cas, les deux formes, féminine et masculine, vont «cohabiter ». En pratique, cela va donner des formes longues typographiquement séquencées qui laissent apparaître, comme par transparence, les formes courtes. C'est une sorte de segmentation qui met en lumière la structuration morphologique du mot. Pour la réaliser, les scripteures et scripteurs peuvent utiliser quatre outils typographiques : le tiret, le point, le point médian ou la majuscule.

Pour les termes dont le radical reste inchangé, les quatre typographies peuvent être employées pour séparer/ajouter la forme longue féminine quand celle-ci est en $-e$ : regroupé-e; manifestantE; auteur.e; certain.e.

Si la flexion de genre entraîne une modification autre que la présence ou l'absence du $-e$, par exemple une modification à la frontière droite du radical, on peut trouver cette modification intégrée au marquage : gardien-ne-s; gentillEs.

Le radical peut être présenté au masculin, qui est alors perçu comme la forme de base : émeutier-e-s; émeutierEs.

Ou bien avec le radical dans sa forme féminine, avec une perception du féminin comme forme de base : familièr-e-s; guerrièrE.

Si le mot est au pluriel, la marque du féminin peut être encadrée de tiret ou de point (ligne de base ou médian). Au singulier, elle en sera précédée ou bien mise seule en majuscule, à la manière d'une incise. On agit donc là uniquement sur la marque de genre : masqué-e-s.

Le pluriel peut être également directement apposé sur le féminin. Dans ce cas, cela signifie que c'est toute transformation de la forme de base qui est signalée, à moins qu'il ne s'agisse d'une volonté de ne pas encombrer la lecture. C'est généralement le cas, lorsque le pluriel est invariable au masculin : divers.es; misES.

Il arrive également que la forme masculine disparaisse, laissant la forme féminine, tout de même marquée afin de noter la volonté de travail sur le genre : nombreuSEs.

Il ne s'agit pas d'une utilisation du féminin générique, puisqu'un marquage

4. Ce fonctionnement étant majoritaire mais pas absolu. Certaines alternances ont le masculin comme forme longue : balle/ballon, aile / aileron, etc. 
typographique est réalisé, mais bien de double marquage de genre. Ici, on peut supposer que c'est l'oral qui influence le choix d'un marquage linéaire plutôt qu'une alternance (nombreux/ses par exemple).

Le maintien de la linéarité peut également se réaliser par la juxtaposition des lexèmes. Les lexèmes peuvent alors être juxtaposés de manière syntaxique ou typographique.

Le doublet lexical est la stratégie syntaxique qui demande le moins d'implications typographiques et morphologiques. Bien qu'allongeant la lecture, ce procédé permet d'éviter les innovations typographiques et les ruptures visuelles. Dans ce cas, les conjonctions de coordination et et ou ainsi que la virgule sont utilisées : casseurs et casseuses; il ou elle; les travailleurs, les travailleuses.

C'est parfois l'ensemble du syntagme nominal qui est repris (déterminant et substantif), parfois uniquement le substantif qui connaît la variation. De plus le doublet permet de mettre sur un même plan des équivalents sémantiques qui n'ont pas de base morphologique commune : tou-te-s les autres frères et sœurs.

Cette utilisation offre une certaine souplesse puisqu'elle permet un emploi générique avec et ou la virgule qui englobe les deux genres, mais laisse aussi la particularisation possible avec ou.

La juxtaposition des lexèmes peut également recourir à une stratégie typographique, avec le tiret et le slash : haineux-haineuse ; ils-elles; vieux/vieilles; il/elle.

\section{Alternance}

Pour une grande part du lexique, le genre fonctionne par alternance, c'est-àdire que le genre est porté par un suffixe, différent au féminin et au masculin. Le double marquage de genre consiste alors à présenter les deux formes l'une à la suite de l'autre (le plus souvent masculin puis féminin, et plus rarement féminin puis masculin). La coprésence de formes habituellement en alternance brise alors la linéarité de la langue, comme un forçage paradigmatique de l'axe syntagmatique. C'est dans ces cas qu'on va trouver le plus de possibilités typographiques - point, point médian, tiret, slash, majuscule, amalgame5 : nombreux.ses; radicaux-ales; libérateurs-trices; traducteurs/trices; auteurSEs; captifVEs; chômeureuses; traducteurices; toustes.

Selon l'enchaînement phonologique créé par la juxtaposition, certaines de ces formes seront réemployées à l'oral (généralement, celles qui sont rédigeables sans marquage typographique).

Parfois, toute la terminaison n'est pas reprise, et seulement quelques lettres changent : courageux/ses; cambrioleuses/rs.

5. Par amalgame, j'entends la juxtaposition des suffixes masculin et féminin sans marque typographique. 
Hormis pour les amalgames, le pluriel sera systématiquement marqué sur chacun des suffixes.

\section{Genrage des épicènes}

Un dernier type de double marquage consiste à intervenir sur les épicènes. II s'agit là de sur-marquer afin de montrer une volonté d'intervention, y compris lorsqu'il n'y a pas de variation de genre. Ce procédé est minoritaire dans le corpus : sept des dix-sept textes montrent de telles occurrences, mais à l'exception de deux textes qui le pratiquent régulièrement (double marquage de 50\% à 100\% de la totalité des épicènes du texte), cette pratique reste marginale, y compris dans les textes qui l'emploient ( $2 \%$ à $24 \%$ des épicènes touchés) (Abbou, 2011). Il est réalisé à l'aide de la majuscule et du souligné : jeunEs ; vigilE; efficaces; sauvages.

On peut donc récapituler les usages typographiques dans le tableau suivant :

Tableau 1. Emploi du marquage typographique selon les fonctionnements linguistiques du genre

\begin{tabular}{l|c|c|c|c}
\hline & $\begin{array}{c}\text { Cohabitation } \\
\text { linéaire }\end{array}$ & $\begin{array}{c}\text { Juxtaposition } \\
\text { de lexèmes }\end{array}$ & Alternance & Surmarquage \\
\hline Tiret & $\mathrm{X}$ & $\mathrm{X}$ & $\mathrm{X}$ & \\
\hline Point ligne de base & $\mathrm{X}$ & & $\mathrm{X}$ & \\
\hline Point médian & $\mathrm{X}$ & & $\mathrm{X}$ & \\
\hline Majuscule & $\mathrm{X}$ & & $\mathrm{X}$ & $\mathrm{X}$ \\
\hline Slash & & $\mathrm{X}$ & $\mathrm{X}$ & \\
\hline Conj. coordination & & $\mathrm{X}$ & & \\
\hline Virgule & & $\mathrm{X}$ & & \\
\hline Amalgame & & & $\mathrm{X}$ & \\
\hline Souligné & & & $\mathrm{X}$ & $\mathrm{X}$ \\
\hline
\end{tabular}

Chaque texte est donc confronté à l'alternance et à la linéarité du genre et, plusieurs procédés sont disponibles pour chaque fonctionnement. Les textes présentent alors un panaché de procédés (typo)graphiques. Cependant, on voit généralement se dégager pour chaque texte un emploi typographique majoritaire. Le tableau ci-dessous (tableau 2), qui présente le procédé majoritaire utilisé pour chaque texte, montre que le tiret et la majuscule sont les plus usités ${ }^{6}$. Cela n'est pas étonnant pour le tiret, car c'est le seul procédé qui permet

6. Le corpus a été collecté entre 2007 et 2010 . Il semble qu'aujourd'hui la majuscule ne soit plus si majoritaire et que le point soit de plus en plus utilisé. L’astérisque apparaît également plus fréquemment. Une étude sur un corpus plus contemporain permettrait de préciser cette évolution des pratiques. 
le marquage des deux fonctionnements du genre à l'écrit : cohabitation-juxtaposition et alternance. Il faut également noter que quasiment tous les textes ont recours à la cohabitation des lexèmes (noté dans la colonne «Doublets»). La dernière colonne indique si les formes en alternance sont présentées dans l'ordre masculin/féminin (M/F) ou bien féminin/masculin (F/M).

Tableau 2. Formes typographiques du double marquage de genre dans le corpus

\begin{tabular}{l|c|l|c|l}
\hline & Double marque & Typo & Doublets & Alternance M/F ou F/M \\
\hline ALF & oui & maj. 63,5\% & oui & Stable $83 \% \mathrm{M} / \mathrm{F}$ \\
\hline MC & oui & maj. 90,3\% & oui & Aléatoire \\
\hline Diap. & oui & tiret $100 \%$ & oui & $\varnothing$ \\
\hline AD & oui & maj. 92,3\% & oui & Stable $100 \% \mathrm{M} / \mathrm{F}$ \\
\hline G1 & oui & tiret $68,4 \%$ & oui & Stable F/M \\
\hline G2 & non & & & \\
\hline G3 & oui & tiret $100 \%$ & non & $\varnothing$ \\
\hline G4 & oui & maj. 83,3\% & oui & Stable $80 \% \mathrm{M} / \mathrm{F}$ \\
\hline G5 & oui & maj. $76,1 \%$ & oui & Stable $78 \% \mathrm{~F} / \mathrm{M}$ \\
\hline G6 & Non & & & \\
\hline G7 & oui & tiret $86,6 \%$ & oui & Aléatoire \\
\hline BB1 & oui & tiret $91,2 \%$ & oui & Stable $85,7 \% \mathrm{M} / \mathrm{F}$ \\
\hline BB2 & oui & tiret $83,3 \%$ & oui & Stable F/M \\
\hline BB3 & oui & tiret $64 \%$ & oui & Stable F/M \\
\hline BB4 & oui & tiret $100 \%$ & non & $\varnothing$ \\
\hline & & & \multicolumn{2}{|l}{}
\end{tabular}

\section{Typographie, politique, linguistique}

On entend fréquemment que la parenthèse est «moche» et minimise les femmes, la majuscule les valorise, le tiret alourdit le texte, le point est discret, etc. (Abbou, 2011). Pour comprendre ce qui guide le choix des procédés, j'ai réalisé des entretiens avec quatre scripteures et scripteurs qui emploient le double marquage du genre, dans lesquels ils discutent des différents procédés typographiques disponibles et justifient leur choix 7 . Les arguments sont de quatre types : typographique, technique, politique, esthétique. Ils s’interrogent ainsi sur les enjeux de lisibilité ainsi que sur la possibilité d'une normalisation

7. Le corpus contient 5 h 20 mn d'enregistrement. Il est constitué de 63000 mots. Il est disponible en ligne sur Ortolang : dépôt et diffusion / Banque de données parole et langage : 〈http://sldr. org/sldroo0714> (consulté le 19 janvier 2017) 
des formes. La dimension esthétique est également convoquée, tout comme la difficulté ou l'aisance technique. Enfin, une sémiotique de la typographie du genre est déployée. Les procédés dont parlent ces locuteurs et locutrices sont les mêmes que nous avons relevés dans le corpus écrit, à l'exception du souligné qui était très marginal et qui n'est pas mentionné dans les entretiens.

\section{Lisibilité}

En typographie, la notion de gris typographique désigne l'impression produite sur l'œil par la répartition des blancs et des caractères dans un texte. De nombreux paramètres entrent en compte dans la composition du gris typographique : l'interlignage (espacement entre les lignes), la chasse et l'approche des caractères (espacement entre les lettres), la densité des lignes (nombre de caractères par ligne) et leur justification (justifié, en drapeau, etc.), la taille des marges, le respect des espaces insécables, mais aussi le suivi de la ligne de base, la ligne inférieure sur laquelle reposent les lettres sans jambage (comme $a, z, s, u$ ). Autant d'éléments qui ont longtemps relevé d'un savoir-faire typographique, et qui sont aujourd'hui gérés automatiquement par les traitements de texte. Un mauvais gris typographique fera apparaître des lézardes, c'est-à-dire un effet de lignes blanches verticales entrainé par de mauvais espacements, et plus généralement ce qu'on appelle des accidents de lecture. Voici un exemple de deux gris typographiques, réglé et non-réglé (Fig. 2) :

Fig. 2. Exemple de deux gris typographiques

Les différents facteurs qui influencent le gris typographique et son homogénéité sont la police (ou " fonte "), le corps (taille), l'interlignage, la graisse, I'utilisation de majuscules, la justification et l'approche entre les caractères (interlettrage).

\begin{abstract}
Les différents facteurs qui influencent le gris typographique et son homogénété sont la police (ou " fonte "), le corps (taille), l'interfignage, la graisse, l'utilisation de majuscules, la justification et l'approche entre les caractères.
\end{abstract}

Sans besoin de connaissances typographiques, notre œil « entre » plus facilement dans un texte si le gris est harmonieux (celui de gauche dans la figure ci-dessus). Or, le double marquage de genre va entrainer une perturbation du gris typographique : la plupart des signes utilisés ne reposent pas sur la ligne de base (tiret, slash, parenthèses, etc.), perturbent la chasse et la densité des lignes, augmentent les blancs et les espaces. Le critère de lisibilité est donc très souvent convoqué pour souligner la nécessité que le texte reste visuellement cohérent. Le slash, la majuscule et les masculin/féminin aléatoires sont reconnus comme gênant la lisibilité alors que le tiret et le point médian la favorisent, la discrétion du point et le caractère dédié du point médian sont citées :

G37 : l'idée [...] c'est que ça HEURTE pas la lecture [...] mettre du féminin et du masculin aléatoirement [...] je trouve je trouve que ça rend la lecture plus difficile + l’idée [...] c'est pas de euh de bloquer la lecture. 
G44 : la capitale [...] ça te perd plus le regard.

$\mathrm{G} 68$ : le tiret c'est plus long du coup ça $\mathrm{f}$ - + freine la lecture et tandis que le point il est il est plus discret.

G79: quand tu te retrouves dans un texte avec des il elle des il euh slash elle euh toutes les deux lignes [...] c'est pénible à la lecture.

G233 : ces créations de nouveaux mots [...] quand il y a des paragraphes avec plein de trucs comme ça moi je trouve ça chiant.

C368: quand c'est mis comme ça peut-être parce qu'aussi c'est sur la même ligne sur le même euh les lettres elles continuent tu as pas un truc qui qui accroche autant euh l'œil quoi à la lecture.

C375: les phrases où j'ai le plus de mal là ces derniers temps c'était des phrases où justement tu avais le la les ou je sais pas quoi + et puis après tu as oh et puis oh c'est quand qu'elle se finit la phrase quoi.

T9: c'est pas un tiret parce que je trouve que ça fait euh c'est illisible c'est juste un petit point euh le plus discret possible.

Il y a donc un équilibre à tenir entre le fait de visibiliser le marquage tout en maintenant une lisibilité maximum.

\section{Normalisation/Perturbation}

Cette question de la lisibilité rejoint également celle de l'accessibilité. Là encore les scripteuses et les scripteurs sont pris dans une tension entre perturbation et normalisation. D'une part, la logique perturbatrice du double marquage vise à attirer l'attention sur la question du genre, mais d'autre part la régularité est recherchée pour développer un marquage le plus harmonieux possible, lisible par tous.

Dans les faits, les entretiens révèlent une plus grande attention à la régularité que ce que l'on peut trouver dans les textes, mais toutes les personnes interrogées ne partagent pas la même posture. Deux des locuteurs et locutrices posent la variabilité des formes, l'instabilité des procédés comme un problème :

G37 : mettre du féminin et du masculin aléatoirement alors en même temps peutêtre ça interpelle plus [...] je trouve que ça rend la lecture plus difficile.

G44: des règles de féminisation il y en a plein [...] il y a une personne pour chaque règle et les textes des fois je trouve qu'ils sont féminisés n'importe comment.

G234 : VRAIMENT cette histoire de lisibilité euh de pas compliquer la lecture ENFIN de pas la compliquer pas OUTRE MESURE c'est-à-dire c'est important de visibiliser le truc mais ça doit pas euh c'est-à-dire je pense pas autant.

G236 : [i ll e s, id2zEl@Es]++ ça ça me gêne c'est un truc ça tombe pas sous le sens donc ça ça s'adresse vraiment qu'à un cercle d'initiés surtout qu'en plus ces texteslà + tu as pas de glossaire la plupart du temps c'est pas expliqué + donc soit tu es un habitué tu es une habituée du truc et tu comprends.

C330 : entre le $[e, 2]$ majuscule le tiret la le le slash le machin le truc + tu vois à 
chaque fois c'est encore une autre euh [...] c'est-à-dire que pour que ça devienne vraiment une un code comme une habitude si à la limite il y avait une euh justement comme il y a des règles typographiques ouais un standard que là c'est pas le cas à chaque fois que tu vas tomber sur un texte.

Alors qu'un troisième locuteur considère que la normalisation n'est pas nécessaire :

T388 : je sais pas si c'est la priorité de se de trop se prendre la tête sur le sur les sur le texte sur les phrases sur les mots la féminisation elle est comprise que tu mettes un grand $[e, 2]$ un tiret une parenthèse [...] un point c'est je pense que c'est compris pour l'instant je vois pas l'intérêt et puis ceux qui se mettent à créer des nouveaux mots j'ai je trouve que c'est pas mal [...] ils sont compréhensibles leurs nouveaux mots et donc ça c'est voilà ça suffit tant que ça devient compréhensible je pense qu'on a pas besoin de tout normer.

\section{Réalisation technique}

Certains locuteurs soulignent la difficulté technique (informatique) pour réaliser certaines formes comme le slash ou le point médian :

G66 : un point mais un point qui est pas le point classique de fin de phrase c'est un point qui est surélevé c'est-à-dire qui est euh alors c'est technique le point il est en b-il est pas sur la ligne euh de base de la phrase le point il est il est au milieu de la hauteur de la lettre donc ça c'est un truc que euh que je trouve pas mal bon qui demande une manip après techniquement qui demande une manip euh supplémentaire.

C352: bon euh peut-être sans compter le fait que euh je suis une pas une pro euh + de l'ordi quoi tu vois mais + donc slash faut mettre alt @.

E325 : je sais pas je bidouille + euh je crois spontanément c'est trop compliqué donc je mettrai radicales et radicaux.

\section{Esthétique}

Quatrième critère évoqué dans le choix des procédés, l'esthétique est mobilisée. On peut parler ici d'eugraphie et de cacographie ${ }^{8}$. Ce critère sera dévalué, donné comme un critère non pertinent, mais toujours présent. Les procédés cacographiques mentionnés sont le tiret et les amalgames, qui sont qualifiés par les locuteurs de «nouveaux mots»:

G64: le tiret [...] au niveau lisibilité ça va au niveau esthétique euh.

G229 : moi il y a des euh par exemple un truc vraiment au niveau féminisation que je trouve mais AFFREUX dans la lecture + c'est euh les nouveaux mots c'est tu crées des mots euh ah en plus c'est c'est pas que je suis pas je suis je suis nul en

8. Pour calquer l'opposition - pour l'oralité - d'euphonie et de cacophonie. 
orthographe en grammaire enfin le euh + c'est pas un rapport euh à l'académie française et tout ça mais.

T114 : quelqu'un dans le groupe fait euh avec des tirets moi j'aime pas c'est c'est peut-être qu'esthétique hein c'est peut-être con bon enfin.

\section{Sémiotique politique de la typographie}

Enfin, la question du sens des signes typographiques est posée, notamment la survalorisation (majuscule) et la dévalorisation (parenthèse), mais également, l'identification de pratiques typographiques à certains milieux politiques ou à certaines époques :

G32 : c'est limité euh à certains milieux euh militants euh issus du féminisme après je trouve que c'est en train de se + de s'étendre à d'autres milieux militants beaucoup plus + enfin beaucoup plus larges je le vois euh mettons ces dernières années [...] par exemple chez des a.sso.cia.tions peut être euh a priori moins radicales qui commencent à mettre des [e, 2] capitale [...] même voire plus institutionnels même certaines [o n g, oEnZe] commencent à + et même des associations féministes euh anciennes qui féminisaient pas leurs textes.

G43 : la parenthèse c'est [...] en général tu mets entre parenthèses un complément d'information [...] après la capitale c'est une survalorisation.

G63 : la parenthèse ça me semble être euh donc politiquement là justement avoir un un sens qui me plaît pas hein.

G44: souvent on va mettre euh chômeur chômeuse par exemple et puis on va pas mettre euh patron patronne + c'est compliqué ces règles parce qu'à la fois c'est c'est un mélange de ce que euh + de nouvelles versions de la grammaire et à la fois un truc politique et parce que des patrons il y a des patronnes aussi + et euh même si il y en a moins puisque ça fait partie [...] des rapports sociaux de sexe de la société mais néanmoins il y en a et du coup ne pas féminiser par exemple moi je trouve ça bizarre + les patrons c'est réac une femme par exemple peut pas être patronne ou que une femme patronne sera forcément différente d'un homme patron.

$C_{352}$ : le $[e, 2]$ majuscule ça commence à me gonfler quand même + ça me fait trop penser à euh c'est beau d'être une femme alors que c'est nul d'être une femme + euh $\mathrm{mh}+$ il faut montrer au co- au monde à quel point on est importante dans la société.

C361: le petit tiret du coup comme il met euh entre tirets quoi c'est pas non plus la parenthèse où là bon tu peux passer outre ouais et non seulement tu insères mais je trouve la majuscule en gros même le mot tu n'y es plus attentif le mot lui-même.

E65 : la parenthèse pour moi c'est un peu associé aux formulaires euh style euh France Télécom ou l'État français qui t'envoie un truc et qui dit cher client chère clienTE et maintenant au lieu de dire cher client chère cliente ils mettent cher client le $[e, 2]$.

E67 : ou la forme officielle OU la forme des gens qui ont pas réfléchi à la question et qui veulent juste être sympa avec les meufs quoi.

E72 : quelqu'un de droite il va pas féminiser ou ou avec des parenthèses quoi. 
T9: je fais un choix très clair c'est pas le grand $[e, 2]$ parce que pour moi ça met en valeur euh un genre par rapport à l'autre c'est c'est presque idiot de dire ça hein mais pour moi ça a quand même de l'importance.

T388: ah oui à une époque ils mettaient beaucoup de parenthèses aussi.

T407 : le [e, 2] majuscule c'est pour moi ça met en avant euh le féminin + ce à quoi on m'a répondu oui mais de toute façon le masculin est TOUJOURS en avant donc ça le met pas plus en avant le féminin c'est + euh ben si ça me dérange parce que c'est euh + ça choque plus.

La parenthèse (qui n’apparaît pas dans le corpus de textes) est rejetée en bloc dans tous les entretiens, comme un procédé qui n'est pas acceptable politiquement. La majuscule est dans l'ensemble refusée (contrairement à ce qu'on trouve dans les textes) car elle est perçue comme valorisant la femme, et non comme questionnant le genre en tant que rapport de pouvoir. Ce sont les seuls procédés qui seront évalués politiquement, et dont le sens typographique fait consensus. Les autres procédés ne sont pas perçus comme véhiculant de sens particulier, comme le montre cet extrait qui pourrait laisser imaginer une problématisation du tiret, mais qui est finalement reformulée :

T11 : tout en montrant que il y a bien euh le féminin qui qui suit le masculin enfin qui a qui est avec pardon.

Mais une locutrice envisage que des procédés puissent devenir politiques :

E301 : le jour où ils diront euh il faut absolument mettre un [e, 2] entre entre guilleentre tirets [...] j'imagine qu'on trouvera un nouveau truc [...] en féminisant nos trucs différemment.

On retrouve dans le tableau suivant (tableau 3), un récapitulatif de ces arguments. Dans l'ensemble, le tiret et le point sont les procédés qui ont la préférence des locuteurs, pour leur discrétion et leur lisibilité, parfois par élimination des autres procédés. La majuscule et la parenthèse sont unanimement rejetées. La première l'est pour son manque de lisibilité, mais elle est surtout évaluée comme une survalorisation du féminin, ce qui pose un problème politique. En effet, la majuscule est perçue comme essentialisant les femmes, à travers une mise en valeur du féminin. Cette valorisation a pu être développée notamment par le féminisme différentialiste, et - conséquemment - ce procédé s'est diffusé dans les cercles les moins radicaux se préoccupant de la question des femmes. Aujourd'hui, alors que la question du genre tend à remplacer la question des femmes, les espaces féministes réaffirment la nécessité d'une lecture antiessentialiste, faisant de la majuscule le marqueur d'un féminisme de la différence, souvent perçu comme dépassé, ou tout au moins, à dépasser.

La seconde au contraire dévalorise, est réductrice, y compris politiquement. Une locutrice l'assimile à la féminisation officielle ou à la féminisation des gens de droite. Un autre y voit un ancien procédé qui n'est plus utilisé. Le 
slash est un second choix, utilisé quand le tiret ou le point sont impossibles, c'est une bidouille qui demande un peu de technique. Cela ne rencontre que partiellement les observations faites sur le corpus écrit, qui, s’il montrait une assez bonne stabilité de l'emploi typographique, présentait principalement des tirets (sept textes) et des majuscules (cinq textes).

Tableau 3-Tableau des procédés typographiques et des critères de sélection

\begin{tabular}{|c|c|c|c|c|c|c|c|c|c|}
\hline & \multicolumn{2}{|c|}{ Slash } & \multicolumn{2}{|c|}{ Point } & \multirow{3}{*}{ Cap } & \multirow{3}{*}{ Parenthèse } & \multirow{3}{*}{ Tiret } & \multirow{3}{*}{$\begin{array}{l}\text { Masc/Fem } \\
\text { aléatoires }\end{array}$} \\
\hline & & Mot & End & Normal & surélevé & & & & \\
\hline \multirow{4}{*}{ 一 } & $\begin{array}{l}\text { Difficulté } \\
\text { technique }\end{array}$ & \multicolumn{2}{|c|}{$\mathrm{X}$} & & $\mathrm{X}$ & & & & \\
\hline & $\begin{array}{l}\text { Pbm } \\
\text { politique / } \\
\text { sémantique }\end{array}$ & & & & & $\mathrm{X}$ & $\mathrm{X}$ & & \\
\hline & $\begin{array}{l}\text { Difficulté de } \\
\text { lisibilité }\end{array}$ & & $\mathrm{X}$ & & & $\mathrm{X}$ & & $\mathrm{X}$ & $\mathrm{X}$ \\
\hline & $\begin{array}{l}\text { Esthétique } \\
\text { - }\end{array}$ & & & & & & & $\mathrm{X}$ & \\
\hline \multirow{2}{*}{$\mathrm{N}$} & Ancien & & & & & & $\mathrm{X}$ & & \\
\hline & indisponible & & & & & & & & \\
\hline \multirow{4}{*}{+} & Lisibilité & & & & $\mathrm{X}$ & & & $\mathrm{X}$ & \\
\hline & Discret & & & $\mathrm{X}$ & $\mathrm{X}$ & & & & \\
\hline & Dédié & & & & $\mathrm{X}$ & & & & \\
\hline & Accessibilité & $\mathrm{X}$ & & & & & & & \\
\hline
\end{tabular}

Il faut enfin noter que, dans de nombreux cas, les formes étudiées sont indicibles. En effet, en français, l'écrit présente la possibilité de briser la linéarité d'un énoncé, ce qui est plus difficile à l'oral. Formes courte et longue et alternance y sont plus facile à perturber. En la matière, c'est donc l'écrit qui va influencer les pratiques orales. Spontanément les locuteurs n'abordent pas la question de l'oral, et c'est sur mes questions qu'ils répondent :

J535: ++ à l'oral tu vas féminiser ou pas?

C540 : pas vraiment c'est assez bizarre c'est assez ambigu +++ c'est un réflexe de pas le faire et c'est c'est un réflexe de comme je le fais pas de le faire mais.

E163: à l'oral à moins de dire euh toutes et tous enfin ce qui est super long.

T233: de temps en tem- ouais parfois.

À cette occasion, presque tous mentionnent une utilisation du féminin générique, alors que ce n'est pas une solution retenue pour l'écrit, tout en la présentant comme un écart : 
$C_{549}$ : les petites euh + libertés entre guillemets que je m'accorde euh + enfin qui sont pas des libertés quoi mais euh [...] vis-à-vis de certains potes mecs je crois que ça pourrait m'arriver si on est je sais pas euh trois meufs et lui enfin lui je sais pas je pense à quelques potes pas beaucoup hein mais de dire ouais les copines.

E106 : par contre ça m'est arrivé euh de féminiser alors que je savais que il y avait que des mecs par exemple.

G140 : alors des fois c'est m- c'est même assez marrant parce que comme c'est un réflexe c'est plus forcément réfléchi du coup des fois je féminise y compris euh + il y a par exemple je suis avec des copains donc que des mecs + et euh et je vais m'adresser à eux je vais féminiser comme si il y avait des mecs et des nanas.

Ces réactions montrent que le double marquage de genre est avant tout une pratique de l'écrit. G. le pointe d'ailleurs avec le critère de l'alphabétisation :

G236 : ça me semble lisible par tout le monde en tout cas pour les gens qui peuvent lire pour les gens qui ont appris à lire + le français + .

\section{Mises en perspective}

\section{La pratique politique du tumulte linguistique}

Malgré le souhait de certaines locutrices et certains locuteurs pour une normalisation des pratiques, l'hétérogénéité des formes présente dans les textes révèle que cette pratique, loin d'être programmatique ou prescriptive, constitue une perturbation linguistique. Il s'agit davantage, pour l'heure, de stratégies et de tendances que d'un système régulier. Cette hétérogénéité relève d'une logique du tumulte (Dorlin, 2005), d'une prolifération des discours (Foucault, 1971). Or la présence de cette perturbation dans l'espace textuel anarchiste n'est pas anodine.

Tout d'abord les anarchistes se sont souvent tenus à proximité de la fabrique de l'écrit : syndicats de correcteurs et imprimerie sont historiquement des lieux remplis d'anarchistes.

Il y a ensuite des conditions matérielles. La brochure politique, qui constitue un pan important de la production discursive anarchiste, est un support privilégié de diffusion des idées et d'expression hors de l'institution. Des textes courts, qui peuvent être écrits sur le vif et circuler rapidement, un média peu coûteux et peu surveillé, à l'anonymisation et à la diffusion faciles. Mais surtout, c'est un support fluide, non-normalisé, au caractère officieux et subversif (Abbou, 2011). Lieu d'expérimentation scripturale non surveillée, les brochures anarchistes sont donc le théâtre idéal pour une nouvelle pratique discursive, farouchement irrégulière. On teste de nouvelles pratiques de la langue et on y interroge les catégories de genre.

Enfin, au-delà du média, je voudrais affirmer que l'anarchisme permet ces expérimentations politico-linguistiques aussi en raison de son articulation 
entre théorie et pratique. Tout comme le langage est depuis longtemps pensé par les féministes comme un lieu où « les normes et les définitions sont remises en questions [et] donc un lieu de résistance» (Sanchez, 2004, p.14), certains points de vue anarchistes insistent sur la nécessité d'échapper aux procédures du discours. Ainsi, mettre le pouvoir en question nécessite de mettre en question le discours. Dans ce but, il est nécessaire de maintenir les pratiques insubordonnées aux discours (au sens de théorie). Vivien Garcia identifie trois éléments susceptibles de ce dégagement : 1) l'usage de média non-institutionnel, 2) une relation particulière à la notion d'auteur, 3) le fait d'ignorer, ou même de refuser la police du discours [philosophique], ici la police de la langue. Cela conduit à faire du discours simultanément la base et la conséquence de la pratique, et donc de comprendre le discours en tant que pratique. Alors, "pouvant se saisir de la philosophie comme de tout un ensemble de savoirs locaux, non conceptuels ou usant de concepts non philosophiques, incapables d'unanimité, [l'anarchisme] dissout les catégories intellectuelles, propose un savoir pratique» (Garcia, 2012). Et la perturbation de genre relève de ce savoir pratique sur la langue et le discours, qui peut éclore dans l'absence de normalisation ou d'expertise.

\section{Rhématisation du genre: un brouillage du rapportémique/étique}

Ce savoir pratique langagier a donc la particularité de dire quelque chose du genre sans que celui-ci soit l'objet du discours. En d'autres termes, on peut agir politiquement sur la marque linguistique du genre au-delà d'un discours sur le genre. Le genre peut être perturbé pour des raisons politiques sans être le thème d'un discours. Il s'agit là d'une rhématisation du genre, qui prend le relais de sa thématisation. Par rhématisation, j'entends ainsi le fait de réaliser typographiquement ou morphosyntaxiquement une intervention sur le genre (par le double marquage notamment) pour des raisons politiques, sans que le texte ne discute de la question du genre. La rhématisation joue ainsi un rôle de sous-texte du genre, y compris dans les discours qui ne se consacrent pas à cette question. Sur les 300 brochures publiées sur le site infokiosques.net au moment de la création de ce corpus, on observe que plus le genre, le féminisme ou la sexualité sont le thème des textes, moins les textes sont doublement marqués. Seulement $55 \%$ des brochures sur le genre le sont. L'une des raisons de cette absence s'explique par le fait qu'une majorité d'auteurs de ces textes sont des femmes. Ainsi, dans un certain nombre de cas, il n'y a pas d'utilisation du masculin du tout. Les textes sont féminisés, au sens littéral. Ils sont écrits au féminin.

Mais une seconde raison apparaît : si l'on veut tenir un discours sur le genre, il est nécessaire de le nommer, de désigner les catégories en jeu afin d'en faire 
la critique. La perturbation des catégories de genre ne peut donc apparaître que lorsque le genre n'est pas le thème du discours, mais le rhème du discours. Le genre devient une sorte de processus prédicatif, qui «conjugue» un discours sur le monde.

Comment se produit alors le passage du rhème au thème dans les textes? Le genre peut être thématisé, et la perturbation du genre abandonnée le temps d'identifier les catégories que l'on critique, et réapparaître sitôt que l'on revient à un autre thème. Dans le corpus écrit, sept textes ne topicalisent pas la question du genre, le rhématisent donc, mais on remarque que la rhématisation (via le double marquage) disparaît dès que des personnes particulières sont désignées : je suis petite et seule; mon ami; je suis un pirate.

Cinq autres textes discutent de la question du genre, c'est-à-dire la thématisent. Les termes référant directement au genre ou à la sexualité sont alors " unimarqués» : vierges; salopes; gouines; pédés; la femme de ma vie.

Le texte intitulé Diaporama: l'apartheid des sexes présente une illustration intéressante des interférences entre thématisation et rhématisation du genre: Nous sommes unisexuel-le-s.

La volonté de sortir du genre refuse ici l'unicité grammaticale et prend le parti de la perturbation où le rhème (double genre) contredit le thème (genre unique).

On voit là clairement la matérialité linguistique être porteuse de signification politique. La transformation est significative seulement si elle est en langue, à la marge du discours. La question du genre est traitée par un focus sur le système linguistique d'opposition du genre qui devient un lieu de subversion. Ce désordre du discours nous entraîne alors, dans le sillage de Marc Angenot, à prendre en compte simultanément le « point de vue formel du texte et [le] point de vue socio-critique de l'idéologie» (1982, p.11) pour observer les échappées aux polices du discours et de la langue. S'emparer de la (typo) graphie permet de multiplier les significations du genre, et d'ajouter de l'hétérogénéité au cœur du signe. C'est donc une saisie politique de la langue.

Cela révèle également une compréhension du langage comme un processus plutôt qu'un système. Cette perturbation est ainsi un brouillage de l'opposition émique/étique. Si le niveau émique est celui de la catégorie, de la langue comme abstraction, comme système, le niveau étique est celui de la réalisation particulière, le champ du discours. En intervenant contextuellement sur la typographie de la morphosyntaxe du genre, les locuteurs et locutrices se situent à l'interface de la langue et du discours, pour nous rappeler que rien n'est en langue qui n'ait d'abord été en discours9 (Benveniste, 1966, p.131). Cette subversion des catégories de genre joue ainsi sur les possibilités

9. Benveniste donne la phrase en latin : «nihil est in lingua quod non prius fuerit in oratione». 
étiques pour faire travailler l'élasticité du syst-ème ${ }^{10}$, en explorant ce que celuici contient de potentialités pour de nouvelles réalisations étiques.

C'est alors le lien entre ce qui est dit et ce qu'il est possible de dire qui est interrogé. Ce champ des possibles est précisément la définition que Eugenio Coseriu donne du système : "un ensemble de possibilités de réalisations» (1973). Louis Hjelmslev, dans le même sens, écrit que «la langue est au langage, ce qu'est la parole à la langue et l'usage à la norme : c'est la réalisation d'un réalisable. Le système du langage est un système de réalisables généraux, et non de réalisables universels » (1971, p.140). Si le système n'est pas la norme, il est cependant une affirmation de la régularité, certes abstraite. Cette abstraction, cette catégorisation par la régularité, est ce qui permet de penser le monde collectivement. Le double marquage de genre est alors une proposition minoritaire de recatégorisation du genre.

La tension entre la dimension systémique de la catégorie grammaticale du genre, et la dimension étique du double marquage de genre apparaît cependant dans les tentatives de l'étendre à des termes ne désignant pas des humains. On en trouve un exemple dans une brochure consacrée à la libération animale, qui critique le rapport hiérarchique entre les espèces animales. Il s'agit de dénoncer une discrimination fondée sur une domination entre les espèces. C'est donc le même schéma que la domination de genre qui est ici dénoncé. Les deux discriminations vont alors s'entrecroiser, et l'auteur choisit d'appliquer la rhématisation du genre aux termes désignant des animaux : rongeurSES; ratonNEslaveurSEs; renardEs; loupVEs; chatTEs sauvages.

L'emploi du double marquage de genre montre un respect de l'exigence antisexiste, mais son application systématisée aux animés relève du respect de la «contrainte antispéciste» : ce qui vaut pour les animés humains vaut aussi pour les animés non humains. Il y a une volonté d'égalisation entre les espèces, en appliquant les mêmes catégorisations aux unes comme aux autres. On pourrait lire cette tentative d'élargissement du double marquage de genre comme une systématisation de cette pratique : si elle est réellement systématique, la marque de genre en effet n'est plus porteuse d'opposition sociale. Mais des irrégularités contredisent cette interprétation. On trouve ainsi dans le même texte : chasseur(SE?)s.

On trouve également une illustration de la tension émique/étique avec l'application de la perturbation du genre à des référents non humains ou abstraits :

- changements et évolutions instantanées, si déroutant-e-s;

- un mélange de races, de genres, de classes et de positions politiques divers-e-s animé-e-s.

Il s'agit ici d'un emploi du double genre comme composante syntaxique permettant d'ordonner la phrase et de faire sens (rattachant [instantanées] à [évolu-

10. J'introduis un tiret ici pour souligner la dimension émique intrinsèque au système de la langue. 
tions], et [déroutant-e-s] au syntagme [changements et évolutions]). C'est à nouveau une proposition de grammaticalisation du double genre, propre à l'écrit. Mais ces réalisations restent extrêmement marginales dans le corpus, et soulignent plutôt les limites d'une compréhension systématique du double genre.

Les formes du genre que l'on trouve dans les brochures anarchistes forment une proposition de double marquage du genre qui se démarque de la féminisation par une volonté de remettre en question la catégorisation de genre ellemême. Propre à l'écrit, cette démarche, qui présente côte-à-côte le masculin et le féminin, montre une hétérogénéité typographique qui répond aux différentes formes que prend le genre en français, principalement par la coprésence de formes habituellement en alternance, qui brise alors la linéarité de la langue et par la réalisation de formes longues séquencées pour permettre de voir apparaître, comme par transparence, les formes courtes.

Au-delà des possibilités graphiques de marquage du genre, ceux et celles qui pratiquent le double marquage de genre dans leurs écrits montrent que les choix typographiques sont en lien avec des questions de lisibilité, d'esthétique et de difficulté technique, mais aussi de normalisation et de sémiotique politique de la typographie.

Cette préoccupation pour les formes écrites du genre dans l'espace textuel anarchiste n'est pas anodine, puisque l'anarchisme cultive une attention subversive au discours, en particulier écrit, ainsi qu'une réflexion sur les rapports de pouvoir, dont fait partie le genre. Le rejet de la systématisation, inscrite dans une culture de l'irrégularité qui refuse de se normaliser, va alors révéler ces pratiques graphiques du genre comme une rhématisation, une possibilité d'ancrer la critique du genre au niveau grammatical, pour prendre le relais du discours, faisant de la langue un lieu de lutte. Cette plongée dans le système linguistique à travers des réalisations toujours contextuelles interroge alors le rapport émique/étique, pour nous amener à penser que, de même que la langue n'est toujours qu'un potentiel réalisable, le genre est une catégorisation potentiellement modifiable, et, surtout, toujours à rejouer. 


\section{Références}

Аввоч Julie, 2011, L'antisexisme linguistique dans les brochures libertaires : pratiques d'écritures et métadiscours, Thèse de doctorat, Sciences du langage, Université Aix-Marseille 1.

ANGENOT Marc, 1982, La parole pamphlétaire : contribution à la typologie des discours modernes, Paris, Payot.

Baider Fabienne, Khaznadar Edwige et Moreau Thérèse, 2007, «Les enjeux de la parité linguistique », Nouvelles questions féministes, vol. 26, n³ 3, p. 4-12.

Benveniste Émile, 1966, Problèmes de linguistique générale 1, Paris, Gallimard.

CAMERon Deborah, 1992, Feminism and linguistic theory, New York, St-Martin's Press.

COSERIU Eugenio, 1973, Teoria del lenguaje y lingüistica general, cinco estudios, Madrid, Gredos.

Dorlin Elsa, 2005, "De l'usage épistémologique et politique des catégories de "sexe” et de "race" dans les études sur le genre », Cahiers du genre, n³9, p. 83-105.

DumaIs Hélène, 2004, "Une pratique quotidienne : la rédaction non sexiste », Désexisation et parité linguistique (ateliers nos 3 et $30 \mathrm{du} 3^{e}$ Colloque international de Recherches féministes francophones), v. Perry éd., p. 57-63 «www.anef.org/wpcontent/uploads/2015/01/Désexisation.pdf〉 (consulté le 10 février 2017).

FoucAult Michel, 1971, L'ordre du discours, Paris, Gallimard.

García Vivien, 2012, "Trouble dans l'ordre du discours», Philosophie de l'anarchie. Théories libertaires, pratiques quotidiennes et ontologie, Jean-Christophe Angaut, Daniel Colson et Mimmo Pucciarelli éd., Lyon, Ateliers de création libertaire, p. $155-168$.

HJelms Lev Louis, 1971, Essais linguistiques, Paris, Éd. de Minuit.

Houdebine-Gravaud Anne-Marie, 1999, «Femmes/langue/féminisation : Une expérience de politique linguistique en France », Nouvelles questions féministes, vol. 20 $\mathrm{n}^{0} 1$, p. 23-51.

JaKOBSOn Roman, 1963, Essais de linguistique générale, Paris, Éd. de Minuit.

LIDDICOAT Anthony J., 2011, «Feminist Language Planning », Current Issues in Language Planning, vol. 12, $\mathrm{n}^{\circ} 1$, p. 1-7.

Michard Claire, 1999, «Humain/femelle : deux poids deux mesures dans la catégorisation de sexe en français », Nouvelles questions féministes, vol. 20, nº 1, p. 53-95.

MOREAU Thérèse, 2001, Écrire les genres : guide romand d'aide à la rédaction administrative et législative épicène, Genève, État de Genève; DF-SPPÉgalité; CLDE.

SANCHEz Dolores, 2004, "La question linguistique et le genre : Paradoxe d'une rencontre ", Désexisation et parité linguistique (ateliers nos 3 et 30 du $3^{e}$ Colloque international de Recherches féministes francophones), V. Perry éd., p.107-115 «www. anef.org/wp-content/uploads/2015/01/Désexisation.pdf〉 (consulté le 10 février 2017). 